\title{
Deposition and passage of transthyretin through the blood-nerve barrier in recipients of familial amyloid polyneuropathy livers
}

\author{
Mónica M Sousa ${ }^{1}$, José Ferrão ${ }^{2}$, Rui Fernandes ${ }^{1}$, António Guimarães ${ }^{3}$, José B Geraldes ${ }^{2}$, \\ Rui Perdigoto ${ }^{2}$, Luís Tomé ${ }^{2}$, Oscar Mota $^{2}$, Luís Negrão ${ }^{4}$, Alexandre Linhares Furtado ${ }^{2}$ \\ and Maria João Saraiva ${ }^{1,5}$ \\ ${ }^{1}$ Molecular Neurobiology, Institute for Cellular and Molecular Biology, Porto, Portugal; ${ }^{2}$ Transplantation \\ Department, University Hospitals of Coimbra, Portugal; ${ }^{3}$ Neuropathology, Hospital Geral de Santo António, \\ Porto, Portugal; ${ }^{4}$ Neurology Department, University Hospitals of Coimbra, Portugal and ${ }^{5}$ ICBAS, University of \\ Porto, Portugal
}

\begin{abstract}
Familial amyloid polyneuropathy (FAP) is characterized by deposition of mutated transthyretin (TTR) in the peripheral nervous system. Prior to amyloid fibrils, nonfibrillar TTR aggregates are deposited inducing oxidative stress with increased nitration (3-NT). As the major source of TTR is the liver, liver transplantation (LT) is used to halt FAP. Given the shortage of liver donors, domino LT (DLT) using FAP livers is performed. The correlation between TTR deposition in the skin and nerve was tested in biopsies from normal individuals, asymptomatic carriers (FAP 0) and FAP patients; in FAP 0, nonfibrillar TTR was observed both in the skin and nerve in the same individuals; in patients, amyloid was detected in both tissues. The occurrence of amyloidosis in recipients of FAP livers was evaluated 1-7 years after DLT: TTR deposition occurred in the skin 3 years after transplantation either as amyloid or aggregates; in one of the recipients, fibrillar TTR was present in the epineurium 6 years after DLT. Deposits were scarce and 3-NT immunostaining was irrelevant. Nerve biopsies from DLT recipients had no FAP-related neuropathy. Our findings suggest that TTR amyloid formation occurs faster than predicted and that TTR of liver origin can cross the blood-nerve barrier. Recipients of FAP livers should be under surveillance for TTR deposition and tissue damage.
\end{abstract}

Laboratory Investigation (2004) 84, 865-873, advance online publication, 3 May doi:10.1038/labinvest.3700107

Keywords: familial amyloid polyneuropathy; transthyretin; peripheral nerve; domino liver transplantation; bloodnerve barrier

Familial amyloid polyneuropathy (FAP) is a neurodegenerative autosomal dominant disorder characterized by the systemic extracellular deposition of mutated transthyretin (TTR) amyloid fibrils throughout the connective tissue, particularly affecting the peripheral nervous system (PNS). ${ }^{1}$ In the PNS of FAP patients, fibrils occur especially in the endoneurium, leading to axonal loss and neurodegeneration..$^{2,3}$ The onset of clinical symptoms generally occurs before the age of 40 years and consists of progressive sensory, motor and autonomic polyneuropathy. Sensory impairment is among the first symptoms, with pain and temperature sensations being the most severely affected; ${ }^{1}$

Correspondence: Professor MJ Saraiva, IBMC- Molecular Neurobiology, Rua Campo Alegre 823, 4150-180 Porto, Portugal.

E-mail: mjsaraiv@ibmc.up.pt

Received 23 December 2003; revised 20 March 2004; accepted 22 March 2004; published online 3 May 2004 motor involvement occurs later causing wasting and weakness. The majority of FAP patients have early autonomic nervous system involvement. Untreated, the disease relentlessly progresses causing severe disability and ultimately death.

Among the point mutations in TTR that promote amyloidogenesis, the most common is a substitution of Val for Met at position 30 of the protein. ${ }^{4}$ The pathway of fibril formation and the mechanisms through which neurodegeneration occurs in FAP are still not fully understood. Cytotoxicity of early nonfibrillar TTR aggregates has been previously found in asymptomatic FAP patients (FAP 0); ${ }^{5,6}$ despite the absence of amyloid fibrils, FAP 0 nerves show signs of neuronal stress presenting increased expression of proinflammatory cytokines, oxidative stress-related molecules and apoptosis. ${ }^{5,6}$

As approximately $90 \%$ of plasma TTR is produced by the liver, ${ }^{7}$ liver transplantation (LT) has been used as a means of abolishing the production of 
most of the amyloidogenic variant TTR. In 1990 the first LT for FAP was performed ${ }^{8}$ and to date, this procedure is the only treatment that appears to halt the progression of the disease. ${ }^{9-11}$ In spite of the progression of the disease being halted, the irreversibility of some neurological lesions seems to persist $^{12}$ although in some cases, symptomatic, autonomic and sensorimotor improvements, namely improved general well-being, walking ability and regain of bladder and bowel functions were reported. ${ }^{13-16}$ It is, however, generally considered that patients should be transplanted as soon as the symptoms start. ${ }^{17,18}$ The long-term outcome of LT as a therapy for FAP remains unknown.

As the shortage of donor livers has been the ratelimiting factor in the expansion of LT, domino liver transplantation (DLT), which was first reported in Portugal in $1995,{ }^{19}$ has been performed worldwide and allows a donor organ to be used for a subsequent graft in a second liver recipient; in this procedure, FAP patients receive and sequentially donate grafts to other recipients. DLT involves specific ethical and technical problems, the most important of which is the graft of a liver producing a pathogenic protein into a recipient individual. Owing to the lack of knowledge relative to the fibrillogenesis pathway and kinetics of deposition of TTR amyloid, the possibility of de novo amyloidosis in recipients of livers from FAP patients remains a serious concern. In most cases, functionally normal livers (excluding the production of mutated TTR) from FAP patients have been used as a source of organs mainly for selected recipients, particularly older subjects in whom the event of de novo amyloidosis is unlikely. In Brazil, recipients of FAP livers have been followed for clinical and electroneuromyographic signs of FAP and for de novo amyloid deposition in the gut, and no signs of de novo amyloid deposits were observed after a mean follow-up of 24 months. ${ }^{20}$

The purpose of this work was to study the presence of de novo amyloid deposition and toxicity in the skin and nerve of recipients of FAP livers up to 7 years after DLT.

\section{Material and methods}

\section{Participants}

Skin biopsies from normal individuals $(n=5)$ and sural nerve and skin biopsies from asymptomatic carriers (FAP $0, n=10$ ) and FAP patients in late stages of disease progression $(n=8)$ were available at the Hospital Geral de Santo António, Porto, Portugal, since this material was obtained as part of the clinical diagnosis and evaluation of polyneuropathy, prior to the current use of less invasive methods. Initial characterization of nerve biopsies included morphometric studies of nerve fiber density and abundance of amyloid deposits.
DLT, as well as biopsy collection from LT patients, are protocols approved by the Ethics Committee of the Transplantation Department, University Hospitals of Coimbra, Portugal and include informed consent; FAP liver donors had typical clinical symptoms, ${ }^{1}$ a family history of FAP, which included the fact that either their mother or father was deceased with FAP with an average of 50 years of age, and were carriers of the TTR Val30Met variant (demonstration of the TTR Val30Met mutation, performed by DNA analysis, is an international requirement for undergoing liver transplantation). A total of 15 FAP patients (four males, mean age 36 (range 31-44) years and 11 females, mean age 35 (range 28-55) years) were subjected to LT from 1995 to 2003. The livers from the FAP patients were sequentially transplanted into 13 male and 2 female recipients (mean age 53 (range 46-61) years) either with chronic liver disease $(n=11)$ or with liver tumors $(n=4)$. DLT recipients had no clinical history or clinical signs of FAP. Four DLT recipients underwent a skin biopsy before the transplant; skin biopsies were collected either up to 2 years after DLT $(n=6)$, between 2 and 5 years $(n=6)$ or 6 years after DLT $(n=3)$. Nerve biopsies were performed in selected recipients who were subjected to DLT for more 5 years. Evaluation of de novo TTR deposition was performed by TTR immunohistochemistry and Congo red staining. ${ }^{21}$ Control skin biopsies were performed in FAP patients $(n=5)$ and non-FAP patients $(n=4)$ submitted to conventional LT 3 years before.

\section{Morphometry}

Morphometric studies were perfomed on sural nerve biopsy tissue fixed in gluteraldehyde $(1.25 \%)$. in $0.1 \mathrm{M}$ cacodylate buffer $\mathrm{pH} 7.4$, postfixed in osmium tetroxide, and embedded in Epon. Quantitation of myelinated fibers (MF) in semithin sections was performed in an area of at least $0.1 \mathrm{~mm}^{2}$ at a magnification of $\times 1000$. Myelinated fibers were counted, their diameters were measured and the density was calculated. Myelinated fibers with a diameter $<6 \mu \mathrm{m}$ were scored as small and fibers with a diameter $\geq 6 \mu \mathrm{m}$ were scored as large. Unmyelinated fibers (UF) were counted from thin sections in an area of at least $0.005 \mathrm{~mm}^{2}$, and their densities were calculated. A scoring system of patient material was based on the morphometry of MFs and UFs: FAP $0(n=5)$, no reduction in the number of fibers (MFs from 11000 to 7000 fibers/ $\mathrm{mm}^{2}$; UFs from 80000 to 30000 fibers $/ \mathrm{mm}^{2}$ ); FAP 1 $(n=4)$, discrete reduction (MFs varied between $<7000$ and 2000 fibers $/ \mathrm{mm}^{2}$; UFs varied between $<30000$ and 4000 fibers $\left./ \mathrm{mm}^{2}\right)$; FAP $2(n=4)$, evident reduction (MFs $<2000$ and 1000 fibers $/ \mathrm{mm}^{2}$; UFs $<4000$ fibers $\left./ \mathrm{mm}^{2}\right)$; and FAP $3(n=3)$, severe reduction (MFs $<1000$ fibers $/ \mathrm{mm}^{2}$; UFs absent). Standard values for control nerves ranged from 
11000 to 7000 fibers $/ \mathrm{mm}^{2}$ for MFs and from 80000 to 30000 fibers $/ \mathrm{mm}^{2}$ for UFs.

\section{Immunohistochemistry}

For immunohistochemistry, paraffin sections were deparaffinated, dehydrated in a modified alcohol series and incubated in blocking buffer (1\% bovine serum albumin, BSA, and $4 \%$ horse serum in PBS) for $30 \mathrm{~min}$ at $37^{\circ} \mathrm{C}$ in a moist chamber. Incubation with primary antibody at the appropriate dilution in blocking buffer was performed overnight at $4^{\circ} \mathrm{C}$. The primary antibodies used were: mouse monoclonal anti-TTR Mab39-44 ${ }^{22}$ and anti-3 nitrotyrosine (Chemicon, 1:1000). Antigen visualization was performed with the biotin-extravidin-peroxidase kit (Sigma), using 3-amino-9-ethyl carbazole (Sigma) as a substrate. On parallel control sections, primary antibody was replaced by blocking buffer. A series of five adjacent slides separated from each other by $25 \mu \mathrm{m}$ was examined for each individual for TTR IHC. Semiquantitative immunohistochemistry (SQIHC) analysis was performed using the Universal Imaging system (NIH) that performs automated particle analysis in a measured area. The results shown represent $\%$ occupied area \pm s.d.

\section{Electron Microscopy and Immunogold TTR Labeling}

Small skin pieces were fixed in $2.5 \%$ gluteraldehyde in PBS for $2 \mathrm{~h}$ and subsequently washed in PBS. Semithin sections $(1 \mu \mathrm{m})$ were cut from eponembedded blocks and stained with toluidine blue. Ultrathin sections $(500 \AA)$ were cut in an ultratome (LK Brumma Nova), mounted on nickel grids, blocked with 1\% BSA in PBS for $30 \mathrm{~min}$ and incubated with rabbit anti-human TTR (DAKO) diluted $1: 100$ in $1 \%$ BSA for $1 \mathrm{~h}$ at room temperature. Control grids were incubated with preabsorbed anti-human TTR. Incubation with anti-rabbit immunoglobulins coupled to $10 \mathrm{~nm}$ gold particles (Amersham) diluted 1:15 in 1\% BSA was performed for $30 \mathrm{~min}$ at room temperature. Grids were then stained with uranyl acetate $(3 \mathrm{~min}$ ) and lead citrate (2 min).

\section{Clinical Evaluation of Neuropathy}

Clinical examination for neuropathic assessment was performed by touch in both feet using a $10 \mathrm{~g}$ monofilament as the mechanical stimulus and hot and cold rods as the thermal stimulus; reflexes were tested with a tendon hammer.

\section{Electromyography}

Needle electromyography (EMG) was performed with a concentric needle electrode. Nerve conduction studies were conducted with a limb tempera- ture above $32^{\circ} \mathrm{C}$. Motor responses were recorded with surface electrodes (belly-tendon montage); the radial (wrist-digit I) and median (wrist-digit III) nerves sensory responses were recorded antidromically through near-nerve needle electrodes after superficial stimulation.

\section{Results}

\section{Skin Biopsy as a Valuable Tool for the Evaluation of TTR Deposition and Cytotoxicity}

Detection of amyloid deposits in FAP nerve biopsies is considered the gold standard for clinical evaluation of amyloid neuropathy. We assessed whether skin biopsies, a minimally invasive procedure, are a sensitive tool to evaluate TTR deposition in FAP. TTR immunohistochemistry and CR staining were performed in the skin of normal individuals $(n=5)$, FAP 0 (asymptomatic) individuals with nonfibrillar TTR deposition in the nerve $(n=9)$ and FAP patients in latter stages of disease progression (FAP 2 and FAP $3, n=8$ ). In normal individuals, TTR was absent and CR staining was negative (Table in Figure 1); in seven out of nine FAP 0 individuals, TTR was deposited in the skin only as nonfibrillar material, negative for CR staining (Table in Figure 1), similar to what was detected on their sural nerve biopsies (the same cases as published in Sousa et $a l^{6}$ ). Skin biopsies from two FAP 0 individuals showed the presence of TTR amyloid fibrils (Table in Figure 1) suggesting that in the skin, amyloid can be detected early and that nonfibrillar TTR deposition constitutes a presymptomatic test. All but two of the eight observed skin biopsies from FAP patients with proven amyloid in the nerve presented TTR deposition in the form of amyloid fibrils (Table in Figure 1) with the characteristic involvement of the lower reticular dermis, sweat glands, hair erector muscle and blood vessels. Curiously, one of these cases did not show evidence of TTR deposition despite being symptomatic for 3 years (Table in Figure 1). These data suggest that in the majority of the FAP patients studied, skin biopsies represent a valuable tool and relate with deposition in the nerve.

We have previously shown that nonfibrillar TTR deposition in the nerve is accompanied by oxidative stress in this tissue as detected by an increase in 3nitrotyrosine (3-NT) staining. ${ }^{6}$ To test if similar mechanisms were occurring in the skin, we performed SQ-IHC for 3-NT in skin biopsies from normal individuals $(n=5)$, FAP 0 patients $(n=9)$ and FAP patients in later stages of disease progression (FAP 2 and $3, n=8$ ). Similar to what was observed in the nerve, there was an approximately three-fold increase in 3-NT epitopes in skins with TTR aggregates, when compared to normal skins (Figure 1, histogram). Interestingly, 3-NT staining was higher when TTR was deposited as nonfibrillar aggregates than when the protein was present in the 


\begin{tabular}{|c|c|c|c|}
\hline individual & nerve & skin (TTR/CR) & clinical condition \\
\hline 1 & normal & - & non-FAP \\
\hline 2 & normal & - & non-FAP \\
\hline 3 & normal & $\cdot$ & non-FAP \\
\hline 4 & normal & - & non-FAP \\
\hline 5 & normal & - & non-FAP \\
\hline 6 & FAP 0 & + & no foll ow up \\
\hline 7 & FAPO & $+\quad-$ & asymptomatic 13 years later \\
\hline 8 & FAPO & $+\quad-$ & asymptomatic 13 years later \\
\hline $\mathbf{9}$ & FAP 0 & $+\quad$ & asymptomatic 12 years later \\
\hline 10 & FAP 0 & $+\quad-$ & symptomatic 12 years later \\
\hline 11 & FAPO & $+\quad-$ & symptomatic 10 years later \\
\hline 12 & FAP 0 & $+\quad-$ & symptomatic 10 years later \\
\hline 13 & FAPO & $+\quad+$ & asymptomatic 22 years later \\
\hline 14 & FAPO & $+\quad+$ & no follow up \\
\hline 15 & FAP 2 & - & 3 years progression \\
\hline 16 & FAP 2 & $+\quad-$ & 3 years progression \\
\hline 17 & FAP 3 & $+\quad+$ & 1 year progression \\
\hline 18 & FAP 2 & $+\quad+$ & 1 year progression \\
\hline 19 & FAP 2 & $+\quad+$ & 3 years progression \\
\hline 20 & FAP 3 & $+\quad+$ & 7 years progression \\
\hline 21 & FAP 3 & $+\quad+$ & 7 years progression \\
\hline 22 & FAP 3 & $+\quad+$ & no follow up \\
\hline
\end{tabular}

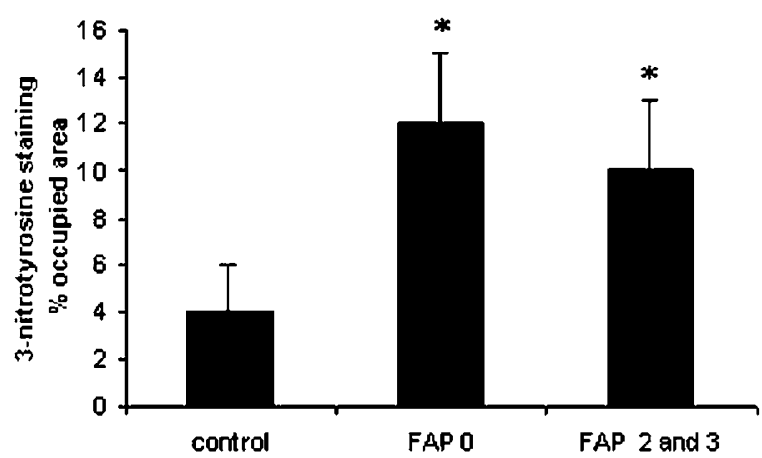

Figure 1 Table: correlation between TTR deposition in the nerve and skin of FAP patients in different stages of disease progression. Histogram: quantitation of immunohistochemical images corresponding to 3-NT immunostaining in control individuals and FAP patients in different stages of disease progression. Data are represented as percent occupied area \pm s.d. $\left({ }^{*} P<0.005\right)$. 
form of amyloid fibrils (Figure 1, histogram). These results suggest that similar to what we have described in the nerve, TTR deposition in the skin of FAP patients is accompanied by oxidative stress.

\section{De Novo Amyloidosis in Recipients of DLT}

Given the fact that TTR deposition in the skin seems to occur early in FAP and to correlate with deposition in the nerve, we analyzed skin biopsies from recipients of FAP livers 1-7 years after DLT. Analysis of skin biopsies performed either prior to DLT $(n=4)$ or in non-FAP individuals undergoing conventional LT $(n=4)$ did not show deposition of TTR (data not shown). FAP patients submitted to conventional LT $(n=5)$ still presented the characteristic TTR amyloid deposition in the skin (Figure 2a, TF). Up to 2 years after DLT, none of the examined skins from recipient individuals presented TTR deposition (Table 1). Between 3-5 years after DLT, deposition was variable and did not correlate with time of transplant; among the two cases of recipients who underwent DLT in the preceding 5 years, one had no deposition whereas in the other amyloid was detected (Table 1). The remaining individuals (4-5 years after DLT) showed only nonfibrillar TTR deposition. Six to seven years after DLT, amyloid deposition was evidenced in all the cases (Table 1). The amount of deposited material was variable among recipients being generally scarce both in nonfibrillar and fibrillar forms, as demonstrated in Figure 2a (TTR staining, arrows, first two rows, DLT $-/+$ and DLT $+/+$ ). One of the recipients in whom the skin biopsy was performed 6 years after DLT presented TTR deposition both in the fibrillar and nonfibrillar form (Figure 2a, third row, DLT $+/+$, arrows) comparable to the characteristic TTR deposition in the skin of FAP patients (Figure 2a, bottom row, TF). The presence of TTR amyloid fibrils in the skin of this DLT recipient was further confirmed by TTR immunoelectronmicroscopy (Figure 2b, arrows); DLT recipients who did not present TTR immunoreactivity were also negative by immunoelectronmicroscopy (data not shown). Given the fact that the amount of deposited

Table 1 Deposition of TTR in skin biopsies performed in recipients of FAP livers 1-7 years after DLT

Years after DLT

TTR/CR

$1(n=3)$

$2(n=3)$

$3(n=1)$

$4(n=2)$

$5(n=1)$

$5(n=1)$

$5(n=1)$

$6(n=2)$

$7(n=1)$
TTR was generally very scarce, dermatological symptoms were absent.

Despite the low amounts of TTR deposition in the skin of DLT recipients (excluding the sole DLT recipient where deposits were comparable to the ones found in FAP), we addressed the possibility that similar to what is observed in FAP patients, TTR deposits might be leading to oxidative stress. 3 -NT IHC in skin biopsies from DLT recipients with fibrillar (DLT $+/+$ ) and nonfibrillar TTR deposition (DLT $-/+$ ), had not increased relative to normal skin biopsies and to non-FAP individuals submitted to conventional LT (Figure 2a, right panels and Figure 2c). FAP patients who underwent conventional LT still presented an increase in 3-NT epitopes in sites related to TTR deposition (Figure 2a, bottom row) typical of nontransplanted FAP patients. In conclusion, the amount of TTR deposition in the majority of the DLT recipients does not seem sufficient to promote an increase in oxidative stress in target organs.

\section{Nerve Pathology in Recipients of DLT}

As DLT recipients presented TTR deposition in the skin, the possibility that TTR deposits existed in the nerve remained to be addressed. Sural nerve biopsies from six recipients of FAP livers who underwent DLT in the preceding 5-7 years were performed. Five of the nerve biopsies had no TTR deposits (Table 2); however, one of the nerve biopsies performed 6 years after DLT disclosed a small deposit of TTR amyloid fibrils in the epineurium (Table 2, Figure 3, arrows). This TTR positive nerve did not reveal any 3-NT immunostaining (Figure 3), similar to what happens in normal nerves, therefore suggesting that the amount of TTR deposition is not sufficient to produce oxidative stress. Morphometry of myelinated and unmyelinated fibers was performed in these nerve biopsies; a minor reduction in the density of myelinated fibers was observed in the majority of the cases, with a more pronounced loss of small diameter fibers in some cases and large diameter fibers in others (Table 2). In all the examined nerve biopsies, the density of unmyelinated fibers was within the normal range for control nerves. The reduction in the number of myelinated fibers is probably not due to the presence of mutated TTR as the majority of the nerves do not show TTR deposition; furthermore, one of the major features of neurodegeneration in FAP consists of the primary loss of unmyelinated rather than myelinated fibers. Clinical examination for putative neuropathic manifestations was performed, but all the DLT recipients presented normal sensory abilities and motor findings such as distal weakness were not present. To further evaluate possible neuropathological changes related with DLT, EMG was performed 5-7 years after transplant. The only DLT recipient who showed TTR deposition 

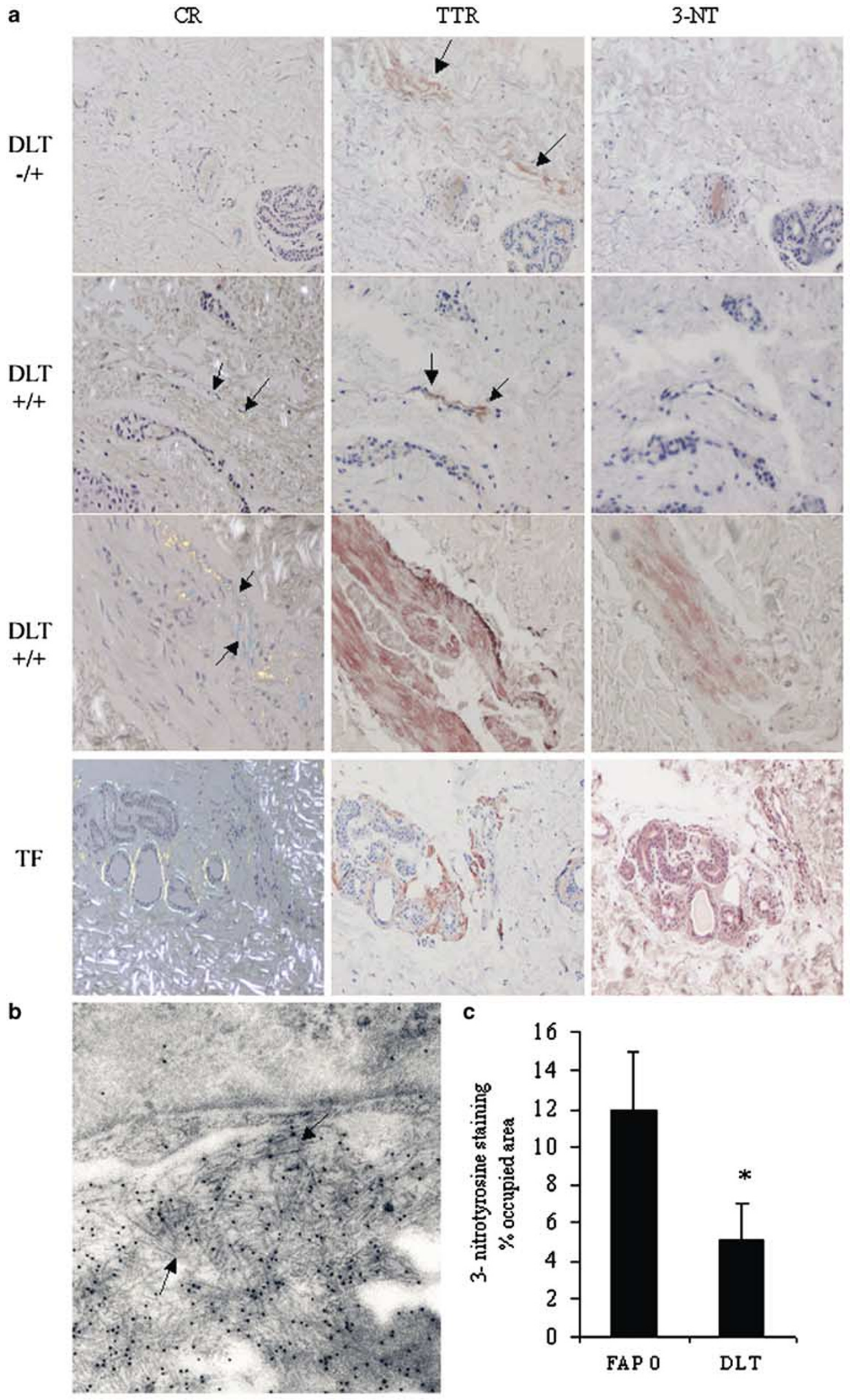
Table 2 Deposition of TTR and morphometric study of nerve biopsies from recipients of FAP livers and EMG evaluation 5-7 years after DLT

\begin{tabular}{llcccccc}
\hline Years post-DLT & Pathology prior DLT & TTR/CR & MF/mm & $\varnothing$ MF lost & UF/mm & EMG prior DLT & EMG at the time of biopsy \\
\hline 5 & Hepatitis + diabetes & -- & 5368 & Large & 39738 & No signs of PNP & Sensory axonal NP \\
5 & Hepatitis & -- & 7331 & Large & 83136 & NA & NA \\
5 & Hepato carcinoma & -- & 3571 & Small & 88330 & NA & No signs of PNP \\
7 & Hepato carcinoma & -- & 3525 & Small & 48340 & NA & No signs of PNP \\
6 & Alcoholic cirrhosis & -- & 4691 & Small & 52951 & No signs of PNP & Sensory-motor changes \\
6 & Neuroendocrine tumor & ++ & 4976 & Small & 44503 & No signs of PNP & No signs of PNP \\
\hline
\end{tabular}

MF, myelinated fiber; UF, unmyelinated fiber; $\varnothing$, diameter; PNP, polyneuropathy; NP, neuropathy; NA, not available.

CR

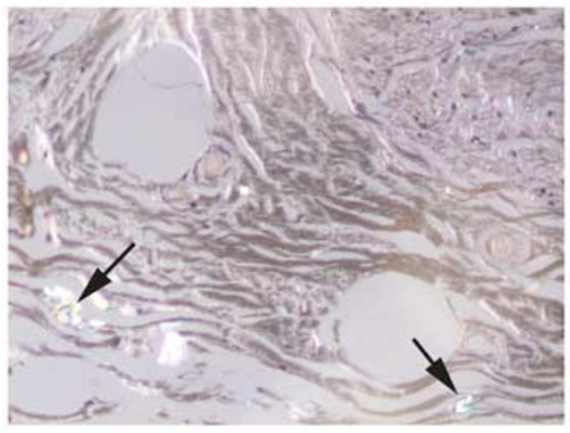

TTR

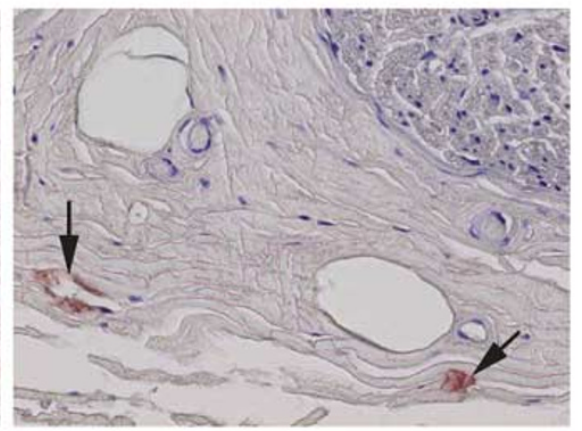

$3-\mathrm{NT}$

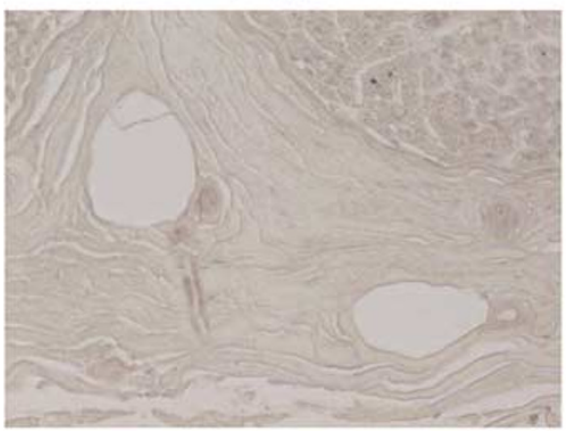

Figure 3 Evaluation of TTR deposition in a nerve biopsy performed 6 years after DLT assessed by CR staining (left panel) and TTR immunohistochemistry (middle panel); oxidative stress evaluated by 3-NT staining (right panel). TTR amyloid fibrils were present in the epineurium without signs of oxidative stress.

in the nerve did not present any signs of polyneuropathy (Table 2), therefore suggesting that the observed deposition of TTR amyloid fibrils in the epineurium was not sufficient to promote neuropathic changes. However, two of the DLT recipients showed signs of polyneuropathy that developed post-transplantation (Table 2). One should however note that in these individuals no TTR deposition was found in the nerve highly suggestive that the observed polyneuropathy was probably caused by other pathologies exhibited by these patients, namely diabetes and alcoholism, in the latter case due to the fact that alcohol intake persisted posttransplantation.

\section{Discussion}

In this report, we show that de novo amyloidosis occurs in the skin and nerve of recipients of FAP livers after DLT. Despite the occurrence of de novo amyloidosis in DLT recipients, the amount of deposited TTR in the majority of the observed cases, both in the skin and in the nerve, was very scarce. Moreover, sites related to TTR deposition did not display signs of oxidative stress probably due to the reduced amount of deposited TTR. At this point, TTR deposits in the DLT recipients do not seem to produce oxidative stress, dermatologic symptoms and FAP-related nerve fiber loss but the long-term effect of DLT remains unknown and one should not rule out the possibility that FAP may develop in long-living DLT recipients.

We started by demonstrating that skin biopsies are a suitable material for the evaluation of TTR deposition, correlating well with TTR deposition in sural nerves. Therefore, the initial assessment of the presence of TTR deposits in skin biopsies from DLT individuals constitutes a valuable tool to assess the possible deposition and deleterious effects of TTR in the nerve after DLT. The DLT recipients reported here showed deposition of TTR in the skin as early as 3 years after DLT, and in the nerve 6 years after DLT. Dermatological symptoms in FAP have occasionally been documented as a consequence of TTR deposition in the skin; multiple atrophic scars

Figure 2 (a) Evaluation of TTR deposition in the skin of recipients of FAP livers by CR staining (left column) and TTR immunohistochemistry (middle column) and the presence of oxidative stress by 3-NT immunohistochemistry (right column). DLT -/ +, CR-negative/TTR-positive recipient; DLT + / + CR-positive/TTR-positive recipient; TF, FAP patient submitted to conventional LT. In DLT recipients, the amount of deposited TTR varied, being generally scarce, and 3-NT had not increased relative to normal skin biopsies; only one case showed TTR deposition comparable to FAP patients. TF presented TTR deposition and 3-NT staining characteristic of FAP patients. (b) Anti-TTR immunoelectronmicroscopy of a skin biopsy performed 3 years after DLT presenting TTR amyloid fibrils. (c) Quantitation of immunohistochemical images corresponding to 3-NT immunostaining in FAP 0 carriers and in recipients of FAP livers (DLT). Data are represented as percent occupied area \pm s.d. $\left({ }^{*} P<0.005\right)$. 
and ulcers on the limbs have been observed in some FAP patients; ${ }^{23}$ nodular cutaneous amyloidosis in a TTR Tyr114His FAP patient has also been described. ${ }^{24}$ However, these occasional dermatological symptoms are only present in the latest stages of disease progression. It should be stressed that in the DLT recipients reported here, the amount of deposited TTR was generally very scarce and therefore dermatological symptoms were not to be expected. A previous study performed in Brazilian recipients of FAP livers did not show any de novo amyloidosis in the gut after a mean follow-up of 24 months probably due to the short time of analysis.

In the TTR Val30Met FAP-associated mutation, the onset of clinical symptoms occurs during adulthood, generally before age 40 years and is fatal in about 10-20 years. The predicted kinetics of amyloid formation is therefore slow and allowed the assumption that DLT recipients would probably not develop TTR deposits shortly after transplant. In the case of the skin, it is possible that aggregated or even fibrillar TTR exists in young asymptomatic carriers prior to major protein deposition in the peripheral nervous system and the occurrence of the first clinical symptoms. In fact, we show that in most asymptomatic TTR Val30Met carriers, TTR is deposited as nonfibrillar aggregates in the skin and in some instances already in the form of amyloid fibrils, reinforcing the idea that skin involvement might represent an early event in the course of the disease.

The fact that recipients of FAP livers show skin TTR deposition as early as 3 years after DLT lead us to raise additionally the possibility that the fibrillogenesis pathway is accelerated in adults either due to the enhancement of mechanisms involved in amyloid formation or due to the downregulation of inhibitory pathways. Given these assumptions, children carrying amyloidogenic-mutated TTR might not show deposition of the protein until adulthood; however, this hypothesis is difficult to test since molecular diagnosis is ethically not possible to perform in children. In asymptomatic TTR Val30Met carriers, TTR is generally present in the nerve in the form of nonfibrillar aggregates, in some cases many years prior to the occurrence of first symptoms. ${ }^{6}$ In one of the DLT recipients whom we report here, TTR amyloid fibrils were present in the nerve six years after transplant favoring the hypothesis that the fibrillogenesis pathway may be preferentially triggered in adults. Organ-specific amyloidogenesis may also be increased upon the process of a transplant; in FAP patients who underwent to conventional LT, although the production of the amyloidogenic-mutated TTR is stopped, cardiac amyloidosis can be exacerbated, probably due to enhanced deposition of wild-type TTR on a template of amyloid derived from variant TTR. ${ }^{25,26}$

The two major sites of TTR synthesis are the liver and the choroid plexus of the brain that are respectively, the sources of TTR found in the plasma and in the cerebrospinal fluid (CSF). ${ }^{7}$ Therefore, the deposited TTR in the PNS of FAP patients may derive from the plasma pool and/or from the CSF pool of the protein. Our report of de novo TTR amyloidosis in recipients of FAP livers demonstrates, however, that in individuals where the liver is the sole source of mutated TTR, the protein is able to deposit in the connective tissue of the epineurium and skin, suggesting that the plasma pool of the protein is sufficient for PNS involvement in FAP. In the nerve, TTR deposition originating from the plasma pool of the protein is probably due to the permeability of the blood-nerve barrier that is effective in slowing but not preventing the entry of TTR into the nerve. One should, however, note that the typical feature of amyloid deposition in the PNS of FAP patients is the presence of amyloid fibrils primarily in the endoneurium. However, the DLT recipient reported here with fibrillar TTR in the nerve showed deposition only in the epineurium, which is generally less affected in typical FAP cases. Therefore, at this point, one cannot exclude that in FAP patients the source of TTR fibrils in the endoneurial space is the CSF pool of the protein.

In FAP nerves, a primary loss of unmyelinated nerve fibers occurs whereas large myelinated fibers are only affected in the latest stages of disease progression. In contrast, we observed that in the case of nerves from recipients of FAP livers, the number of unmyelinated fibers is normal and that a minor reduction only occurs in myelinated fibers, without being associated with clinical manifestations. This observation suggested that the morphometric changes were not related to FAP but were most probably the consequence of other pathologies causing sensory axonopathy, such as diabetes and alcoholism, which were part of the clinical history of some of these individuals. In agreement with this hypothesis, the DLT recipients in whom polyneuropathy developed after liver transplantation had no signs of TTR deposition in the nerve, therefore excluding the possibility of axonopathy caused by TTR deposits. In FAP it has been hypothesized that the loss of sensory and autonomic neurons may function as the pathological background for the preferential degeneration of unmyelinated and small myelinated fibers. ${ }^{27}$ Sural nerve biopsies, on which most of the pathological analysis in FAP has been performed, ${ }^{28}$ represent a restricted portion of the PNS, and it is clearly possible that amyloid deposits in ganglia, or more proximally in nerve trunks, might be responsible for distal nerve fiber loss. In the case of DLT recipients, the fact that the number of unmyelinated fibers is preserved relative to control individuals makes it unlikely that major TTR deposition occurs in the ganglia. It is worth noting the fact that TTR deposition in the epineurium in one of the DLT recipients was not sufficient to promote FAP-related neurodegeneration, as no evidence of polyneuropathy was observed by EMG. 
In conclusion, DLT using FAP livers should continue to be considered an experimental procedure under careful surveillance, and recipients of FAP livers should be followed up using an extensive neurological and pathological examination.

\section{Acknowledgements}

We thank Rosana Moreira (Molecular Neurobiology, IBMC, Porto, Portugal) for tissue processing. This work was supported by Portuguese grants from POCTI Program (Fundação para a Ciência e Tecnologia), Gulbenkian Foundation and SAUDE XXI Program (Health Ministry).

\section{References}

1 Andrade C. A peculiar form of peripheral neuropathy. Familial atypical generalized amyloidosis with special involvement of the peripheral nerves. Brain 1952; 75:408-427.

2 Coimbra A, Andrade C. Familial amyloid polyneuropathy: and electron microscope study of peripheral nerve in five cases. II. Nerve fibril changes. Brain 1971;94:207-212.

3 Dyck PJ, Lambert GH. Dissociated sensation in amyloidosis. Arch Neurol Chicago 1969;20:409-507.

4 Saraiva MJ, Birken S, Costa PP, et al. Amyloid fibril protein in familial amyloidotic polyneuropathy, Portuguese type. Definition of molecular abnormality in transthyretin (prealbumin). J Clin Invest 1984;74: 104-119.

5 Sousa MM, Cardoso I, Fernandes R, et al. Deposition of transthyretin in early stages of familial amyloidotic polyneuropathy: evidence for toxicity of nonfibrillar aggregates. Am J Pathol 2001;159:1993-2000.

6 Sousa MM, Du Yan S, Fernandes R, et al. Familial amyloid polyneuropathy: receptor for advanced glycation end products-dependent triggering of neuronal inflammatory and apoptotic pathways. J Neurosci 2001;21:7576-7586.

7 Maeda S, Mita S, Araki S, et al. Structure and expression of the mutant prealbumin gene associated with familial amyloidotic polyneuropathy. Mol Biol Med 1986;3:329-338.

8 Holmgren G, Steen L, Ekstedt J, et al. Biochemical effect of liver transplantation in two Swedish patients with familial amyloidotic polyneuropathy (FAPmet30). Clin Genet 1991;40:242-246.

9 Takei Y, Ikeda S, Hashikura Y, et al. Partial-liver transplantation to treat familial amyloid polyneuropathy: follow-up of 11 patients. Ann Intern Med 1999;131:592-595.

10 de Carvalho M, Conceição I, Bentes C, et al. Long-term quantitative evaluation of liver transplantation in familial amyloid polyneuropathy (Portuguese V30 M). Amyloid 2002;9:126-133.

11 Suhr OB, Ericzon BG, Friman S. Long-term follow-up of survival of liver transplant recipients with familial amyloid polyneuropathy (Portuguese type). Liver Transpl 2002;8:787-794.
12 Furtado L, Oliveira F, Furtado E, et al. Maximum sharing of cadaver liver grafts composite split and domino liver transplants. Liver Transpl Surg 1999;5: 157-158.

13 Holmgren G, Ericzon BG, Groth CG, et al. Clinical improvement and amyloid regression after liver transplantation in hereditary transthyretin amyloidosis. Lancet 1993;341:1113-1136.

14 Parrilla P, Ramirez P, Bueno FS, et al. Clinical improvement after liver transplantation for type I familial amyloid polyneuropathy. Br J Surg 1995; 82:825-828.

15 Bergethon PR, Sabin TD, Lewis D, et al. Improvement in the polyneuropathy associated with familial amyloid polyneuropathy after liver transplantation. Neurology 1996;47:944-951.

16 Ikeda S, Takei Y, Yanagisawa N, et al. Peripheral nerves regenerated in familial amyloid polyneuropathy after liver transplantation. Ann Intern Med 1997;127:618-620.

17 Adams D, Samuel D, Goulon-Goeau C, et al. The course and prognostic factors of familial amyloid polyneuropathy after liver transplantation. Brain 2000;123:1495-1504.

18 Bittencourt PL, Couto CA, Farias AQ, et al. Results of liver transplantation for familial amyloid polyneuropathy type I in Brazil. Liver Transpl 2002;8: 34-39.

19 Furtado A, Tome L, Oliveira FJ, et al. Sequential liver transplantation. Transplant Proc 1997;29: 467-468.

20 Bittencourt PL, Couto CA, Leitao RM, et al. No evidence of de novo amyloidosis in recipients of domino liver transplantation: 12 to 40 (mean 24) month follow-up. Amyloid 2002;9:194-196.

21 Puchtler H, Sweat F. Congo red as a stain for fluorescence microscopy of amyloid. J Histochem Cytochem 1965;13:693-694.

22 Goldsteins G, Persson H, Andersson K, et al. Exposure of cryptic epitopes on transthyretin only in amyloid and in amyloidogenic mutants. Proc Natl Acad Sci USA 1999;96:3108-3113.

23 Rubinow A, Cohen AS. Skin involvement in familial amyloidotic polyneuropathy. Neurology 1981; 31:1341-1345.

24 Mochizuki H, Kamakura K, Masaki T, et al. Nodular cutaneous amyloidosis and carpal tunnel syndrome due to the amyloidogenic transthyretin His 114 variant. Amyloid 2001;8:105-110.

25 Stangou AJ, Hawkins PN, Heaton ND, et al. Progressive cardiac amyloidosis following liver transplantation for familial amyloid polyneuropathy: implications for amyloid fibrillogenesis. Transplantation 1998;66: 229-233.

26 Golling M, Singer R, Weiss G, et al. Sequential (domino) transplantation of the liver in a transthyretin-50 familial amyloid polyneuropathy. Special reference to cardiological diagnosis and complications. Langenbecks Arch Surg 2000;385:21-26.

27 Thomas PK, King RH. Peripheral nerve changes in amyloid neuropathy. Brain 1974;97:395-406.

28 Sousa MM, Saraiva MJ. Neurodegeneration in familial amyloid polyneuropathy: from pathology tomolecular signaling. Prog Neurobiol 2003;71: 385-400. 